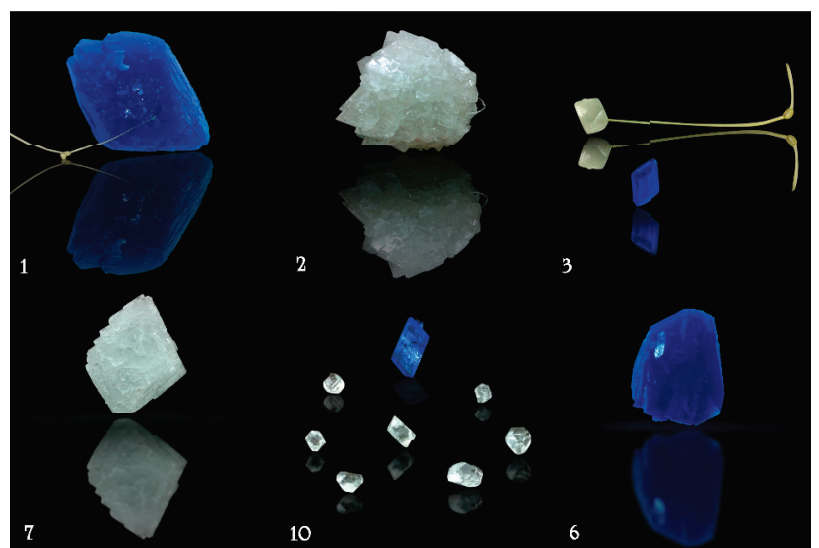

Figure 1. Selected crystals of alum and copper sulfate submitted to the Crystal Growing Competition in Vietnam, edition 2014

Keywords: Crystal growing competition, Vietnam.
MS50. Cultural and historical aspects of crystallography

Chairs: Aleksandar Višnjevac, Petr Bezdička

\section{MS50-P1 Naturally irradiated fluorite as a historic violet pigment: X-ray diffraction and Raman spectroscopic study \\ Petr Bezdička ${ }^{1,2}$, Zdeňka Čermáková, ${ }^{1,2}$, Ivan Němec ${ }^{3}$, Janka Hradilová $^{1}$, David Hradil ${ }^{1,2}$}

1. Academy of Fine Arts in Prague, ALMA laboratory, U Akademie 4, 17022 Prague 7, Czech Republic

2. Institute of Inorganic Chemistry of the ASCR, v.v.i., ALMA laboratory, 25068 Husinec - Řež, Czech Republic

3. Department of Inorganic Chemistry, Faculty of Science, Charles University in Prague, Hlavova 8, 12800 Prague 2, Czech Republic

email: petrb@iic.cas.cz

Naturally irradiated violet fluorite, a cubic $\mathrm{CaF}_{2}$ mineral, is a rare historic pigment. Its documented usage in Europe stretches from ca. 1450 to ca. 1550. The intensely coloured violetish black naturally irradiated fluorite is commonly called antozonite, which is only vaguely defined based on its dark colour and specific odour emanated during grinding. In the published literature, there have been some discrepancies about its physico - chemical properties. Therefore, sixteen samples of antozonite were analysed by X-ray powder diffraction in transmission mode and Raman (micro-) spectroscopy using five different excitation laser wavelengths (445, 532, 633, 780 and $1064 \mathrm{~nm}$ ).

The structural damage of antozonite samples has been assessed by X-ray diffraction and related to their lightness using analysis of image histograms. The XRD study confirmed that the structural disorder caused by natural irradiation may be expressed by broadening of its diffraction lines. The higher is their full-width half-maxima, the lower is the sample's lightness. On the other hand, the reported increase of antozonite's unit cell parameter (and the unit cell volume) is not so straightforward. The studied antozonite samples did not exhibit positive correlation of the parameter and the observed increase of the FWHMs. Still, the sample with the highest full-width half-maxima had also one of the highest values of the unit cell parameter.

Raman spectroscopy revealed specific bands located below $500 \mathrm{~cm}^{-1}$ probably related to radiation-caused defects. Their intensity increased with increasing violet colour saturation, thus providing a specification for antozonite's definition. Spectra excited at 445 and 780 $\mathrm{nm}$ contained also numerous broad bands above $500 \mathrm{~cm}^{-1}$, which seem to be caused by the presence of rare earth elements.

The obtained results have been applied in the analysis of micro-samples of a Late Gothic altarpiece located in an Italian Court in UNESCO city Kutná Hora, Czech 
Republic, which contained exceptionally large grains of deep violet fluorite identified as antozonite.

Keywords: Antozonite, Fluorite, X-ray powder diffraction, Raman spectroscopy
MS50-P2 Pattern zoo part I: on the choices and number of asymmetric units for the 17 plane groups

\author{
Manfred Wildner ${ }^{1}$
}

1. Institut für Mineralogie und Kristallographie, Universität Wien, Althanstr. 14, A-1090 Wien, Austria

email: manfred.wildner@univie.ac.at

The 17 plane groups (PGs, often better known as 'wallpaper groups') and related periodic tilings and patterns played - and still play - an important role in arts and cultural history over the centuries. Even in palaeolithic caves, simple patterns and grids (probably for testing pigments) have been found along with the famous cave paintings. Starting with Kepler's Harmonice Mundi from 1619, they also form an outstanding object of vivid interdisciplinary research, mainly concentrated in the mathematical sciences.

A basic concept of PGs and tilings is the asymmetric unit (ASU) (or 'fundamental domain', 'generating region'). According to the International Tables Vol. A, "An asymmetric unit ... is a (simply connected) smallest closed part ... from which, by application of all symmetry operations ..., the whole of space is filled". It is clear that - except in four PGs - the choice of the ASU, and hence the generated tiling, is not unique. Although this fact is often mentioned in literature, there seem to exist no published enumerations of possible ASUs for the PGs, according to any classification scheme whatsoever.

To enumerate choices of ASUs for the PGs, basic prerequisites are: 1) ASUs (or rather the tile representing an ASU) have to be asymmetric (of course) and by applying symmetry elements the resulting tiling has to fill the plane isohedrally - and thus monohedrally - without overlaps or gaps. From this follows that 2a) mirror planes or rotation axes must not occur inside an ASU; $2 b$ ) mirror planes may form edges of an ASU; 2c) rotation axes may occur on corners (all axes) or edges (2-fold axes only) of an ASU; and 2d) glide planes may occur inside ASUs, but their partial lengths underlie restrictions.

In the present attempt to enumerate, but also to limit, the number of possible ASUs, we will adhere to the following further conditions: 1) we will use only convex straight-line bordered polygons as ASU, i.e. 'Escher-like' ASUs are not considered; 2) to assure that the symmetry of a PG is maintained in the resulting tiling, ASUs are graphically marked as asymmetric (the 'zoo mission'); but, we shall allow for 'symmetrized' ASUs, if the unmarked tiling belongs to a smaller cell or to a supergroup within the same crystal system; 3) we will distinguish cell-confined and non-cell-confined ASUs.

Tilings corresponding to all enumerated ASUs of the 17 PGs will be presented graphically, and, where applicable, references to higher symmetric unmarked tilings will be given.

Keywords: plane groups, asymmetric unit, wallpaper groups, tilings, patterns, polygon tilings, isohedral tilings 